\title{
Steered Molecular Dynamics Study of Inhibitor Binding in the Internal Binding Site in Dehaloperoxidase-Hemoglobin
}

\author{
Zhisen Zhang ${ }^{1}$, Andrew P. Santos ${ }^{3}$, Qing Zhou ${ }^{3}$, Lijun Liang ${ }^{2}$, \\ Qi Wang ${ }^{2}$, Tao $\mathrm{Wu}^{2 *}$, Stefan Franzen ${ }^{2,3^{*}}$
}

1. Research Institute for Soft Matter and Biomimetics, Department of Physics, Xiamen University, 361005 Xiamen, People's Republic of China

2. Soft Matter Research Center and Department of Chemistry, Zhejiang University, 310003 Hangzhou, Peoples Republic of China

3. Department of Chemistry, North Carolina State University, Raleigh, NC 27695

* Co-corresponding authors

Dr. Stefan Franzen

Department of Chemistry

North Carolina State University

Raleigh, NC 27695

Email: Stefan_Franzen@ncsu.edu

Dr. Tao Wu

Soft Matter Research Center

Department of Chemistry

Zhejiang University

Hangzhou, China

Email: tao_wu@zju.edu.cn

Running title: SMD Study of Inhibitor Binding in DHP

Keywords: binding pathway, potential of mean force, protein-ligand interaction,

Michaelis-Menten kinetics, steered molecular dynamics, Jarzynski equality 


\section{Abstract}

The binding free energy of 4-bromophenol (4-BP), an inhibitor that binds in the internal binding site in dehaloperoxidase-hemoglobin (DHP) was calculated using Molecular Dynamics (MD) methods combined with pulling or umbrella sampling. The effects of systematic changes in the pulling speed, pulling force constant and restraint force constant on the calculated potential of mean force (PMF) are presented in this study. The PMFs calculated using steered molecular dynamics (SMD) were validated by umbrella sampling (US) in the strongly restrained regime. A series of restraint force constants ranging from 1000 down to $5 \mathrm{~kJ} /\left(\mathrm{mol} \mathrm{nm}{ }^{2}\right)$ were used in SMD simulations. This range was validated using US, however noting that weaker restraints give rise to a broader sampling of configurations. This comparison was further tested by a pulling simulation conducted without any restraints, which was observed to have a value closest to the experimentally measured free energy for binding of 4-BP to DHP based on ultraviolet-visible (UV-vis) and resonance Raman spectroscopies. The protein-inhibitor system is well suited for fundamental study of free energy calculations because the DHP protein is relatively small and the inhibitor is quite rigid. Simulation configuration structures are compared to the X-ray crystallography structures of the binding site of 4-BP in the distal pocket above the heme 


\section{Introduction}

The binding of an inhibitor, 4-bromophenol (4-BP), in a well-defined binding site in the distal pocket of the multi-functional enzyme dehaloperoxidase-hemoglobin (DHP) from Amphitrite ornata presents an interesting set of biological questions while simultaneously providing a well-defined model system to test computational methods for the calculation of binding free energy.[1,2] The nature of the binding trajectory is of interest since 4-BP must displace a number of amino acid side chains in DHP in order to enter or exit the distal pocket. The modeling of the trajectory using molecular dynamics (MD) simulations has the same features observed in the general class of calculations of drug and ligand binding [3,4] and is particularly closely related to the binding of substrates to cytochrome P450.[5-9] Since 4-BP is relatively rigid, having only the bending and torsions of the hydroxyl group as internal degrees of freedom, the parameters are relatively easily incorporated into the force field for studies of the binding pathway using and the free energy via the potential of mean force (PMF) calculated using steered molecular dynamics (SMD) simulations. This makes the problem similar to the binding of small molecules such as diatomic ligands $\left(\mathrm{CO}, \mathrm{NO}, \mathrm{O}_{2}\right)$, indazole or nicotinic acid, which have also been studied using similar 
methods. [10-14] However, the inhibitor, 4-BP, is significantly larger than a diatomic molecule and therefore the results have more relevance for binding free energy calculations in drug-target or ligand-receptor systems. The calculations the free energy of binding using non-equilibrium methods has been demonstrated using a variety of systems, many of them with far greater complexity that the DHP + 4-BP combination. $[5,6,11,15-20]$ When the ligand has significant internal degrees of freedom, such as in peptide-protein or protein-protein binding, correction terms must be applied to thermodynamic integration used to extract the free energy from the simulated trajectory. [21-24] By contrast the DHP + 4-BP system consists of a small rigid molecule that nonetheless has a well-defined internal binding pocket based on X-ray crystal structures (Figure 1). [1, 25-33] In this study the restraints applied to the DHP protein are systematically reduced and ultimately compared to a restraint-free calculation. The systematic approach reveals a trade-off in the large magnitude of fluctuations in the calculated free energy and the increase in tractability as DHP is more restrained. [34]

The selection of an appropriate free energy calculation method is important for modeling the DHP + 4-BP binding process, and can affect the calculated binding energy. Several methods are present in the literature that permit the calculation free energy differences from equilibrium MD simulation trajectories, such as thermodynamic integration, free energy perturbation (FEP), [4] and umbrella sampling (US) employing the weighted histogram analysis method (WHAM).[35] 
These equilibrium methods can provide an accurate estimation of protein-ligand binding affinities, but they are only applicable if the system is sufficiently rigid so that the various stages of the trajectory have comparable geometries. An alternative non-equilibrium method based on the Jarzynski equality [36, 37], the so-called "fast growth" method, [38] has been developed for free energy calculations as well. The Jarzynski equality has been employed to extract free energy profiles from repeated non-equilibrium pulling trajectories.[39-45] The non-equilibrium SMD method is considered less accurate than equilibrium US methods. [15, 46, 47] However, there are two advantages of the non-equilibrium method in free energy calculations compared to equilibrium methods: i) Non-equilibrium binding (or unbinding) simulations can be conducted for any value of the restraint force constant; ii) The method permits a relatively rapid test of different binding trajectories, which is relevant to the mechanism of protein-ligand interactions. [12, 48-50]

The advantages of the SMD method can be used to study DHP ligand binding. DHP is a multifunctional protein, which has documented functions as a globin (oxygen transport), peroxidase (electron transfer), peroxygenase (oxygen atom transfer) and an oxidase (oxidation by $\mathrm{O}_{2}$ ).[51] The peroxygenase function has similarity with cytochrome $\mathrm{P} 450$ since both involve $\mathrm{O}$ atom transfer in their mechanism. The difference is that the $\mathrm{O}$ atom in $\mathrm{P} 450$ comes from bound $\mathrm{O}_{2}$, while that in DHP comes from bound $\mathrm{H}_{2} \mathrm{O}_{2}$. However, the distal pocket of DHP has not been thoroughly investigated as a ligand binding pocket in the way that the binding pocket of P450 has been studied. In fact, the idea of such an investigation would have been 
considered impossible prior to the recent discovery that DHP has multiple internal binding sites, $[52,53]$ which makes its distal pocket more like the substrate binding pocket of a P450 than a typical globin. The paths for entry and exit of small molecules have been demonstrated based on the cytochrome P450 model system.[5-9, 54] In DHP, the inhibitor, 4-BP, is completely enveloped by the protein in the bound state just like substrates in P450. $[1,25]$ However, the binding of 4-BP appears to be quite specific since the para bromine atom of $4-\mathrm{BP}$ is stabilized by a protein cavity $[55,56]$ and the hydroxyl group forms a hydrogen bond with the heme propionates. Since the trajectory of the inhibitor as it exits the distal pocket is relatively short in DHP, SMD is an appropriate method to test the relevance of specific exit pathways. The conclusions were validated using Umbrella Sampling (US) and Random Accelerated Molecular Dynamics (RAMD). [7-9]

The contemporary idea of a tunnel for small molecule entry and exit trajectories derives from the early simulations using $\mathrm{CO}$ in myoglobin. Protein channels or tunnels have been identified in DHP based on the X-ray crystal structure coordinates (using programs CAVER[57] and MOLE.[58]) and we have used this approach to understand the possible ligand trajectories in DHP. [59] Tunnels have been used to study a range of problems based on non-equilibrium simulations including discrimination between $\mathrm{NO}$ and $\mathrm{O}_{2}$ in guanylate cyclase,[60] binding of ligands to cytochrome P450.[13] Both of these have relevance for DHP, where 4-BP must enter and exit the protein, but so too must $\mathrm{O}_{2}$ and $\mathrm{H}_{2} \mathrm{O}_{2}$ in order for DHP to carry out its various functions. For more than 30 years, the trajectories of photolyzed carbon 
monoxide (CO) in Sperm whale myoglobin (SWMb) have been studied using MD simulations as a model system for protein dynamics. [61-67] For sufficiently long simulations on contemporary computer clusters, the $\mathrm{CO}$ molecule can be observed to escape from the protein, but 4-BP is not likely to escape on the time scales available in a typical MD simulation $(\sim 1 \mu \mathrm{s})$. On the other hand, 4-BP is well suited for methods that permit calculation of a PMF, SMD and US because it is relatively rigid, thus simplifying the thermodynamic integration is required to extract a free energy that can be compared to experiment.

The goal of the present study has both a practical aspect and a fundamental aspect. From a practical point of view we would like to find a method that will permit us to compare the binding of substrates and inhibitors, since we now have evidence for binding of more than ten different molecules in the distal pocket. DHP functions as a monomer in the coelom of the marine worm, A. ornata, as shown by small angle X-ray scattering. [68] Using this monomer model system and the rigid inhibitor 4-BP, we will determine the trajectory with the smallest work for extraction of 4-BP from the protein, which is the most likely path for ligand exit. Free energy calculations based on either SMD or US use restraints, biasing, or fixed coordinates to eliminate high energy paths that are not physically reasonable. While equilibrium simulations, particularly US are considered to be the most accurate, a number of methods have been used to increase the accuracy of SMD simulations. Methods based on bi-directional pulling have complemented these efforts in an attempt to increase the applicability of the non-equilibrium methods to free energy calculations. [69] A 
renormalization approach has been proposed to permit application of SMD to long-time dynamics on the microsecond time scale, which would increase the sampling and therefore the accuracy of thermodynamic averages.[70] The use of local sampling implemented using conformational freezing has been considered as a method to enhance the accuracy of the calculated PMF. [71] Biasing potentials have been implemented that restrict the conformation of ligands to their bound state. [72] Moreover, hybrid MD using a combination of docking and SMD with a scoring function has been proposed to solve these problems for drug delivery applications. [73] In this report, we have used SMD as a method to both establish the reaction path for inhibitor binding and to calculate the free energy. We have addressed the accuracy of the method by comparison with US and by a systematic study of the effect of restraints. Reasonable agreement with experiment is obtained when correction for the restraints are applied.

\section{Methods}

\section{Molecular Dynamics Simulations}

All the simulations were performed in the NVT ensemble at a temperature of 298 K. All the MD simulations were performed using the GROMACS package (version 4.5.2). [74, 75] The DHP and 4-bromophenol, the DHP-inhibitor complex, were modeled with the all-atom CHARMM27[64] forcefield and water was modeled using the TIP3P[76] forcefield. The initial coordinates of the DHP-inhibitor complex were obtained from the protein data bank (PDB code: 3LB2).[1]. A DHP monomer 
complex was solvated by $\sim 7260$ water molecules in a rectangular solvent box constructed with a $1.0 \mathrm{~nm}$ distance around the protein solute. The system consisted of $\sim 24,000$ particles in a box with $\mathrm{x}, \mathrm{y}$ and $\mathrm{z}$ dimensions of $7.2 \mathrm{~nm}, 5.6 \mathrm{~nm}$, and $6.0 \mathrm{~nm}$, respectively. The protein system would ordinarily have a dimension of $5.2 \mathrm{~nm}$ in the $\mathrm{x}$-direction, and thus the increase by $2.0 \mathrm{~nm}$ was added in the $\mathrm{x}$-direction to prevent any possible collision of 4-BP with the DHP image during the pulling simulation.

The time step was chosen to be 2 fs consistent with use of the SHAKE algorithm.[77, 78] Periodic boundary conditions were applied in all directions. Particle-mesh Ewald (PME) [79] summation was applied for treatment of the long-range coulomb interactions. The cutoff distance was chosen to be $1.3 \mathrm{~nm}$. Initial velocities according to Maxwell distribution corresponding to the desired temperature were used as the starting configuration. In order to approach reasonable sampling each trajectory studied consisted of 100 averages of a $20 \mathrm{~ns}$ pulling simulation or $2 \mu \mathrm{s}$ of total simulation time per averaged trajectory. The Visual Molecular Dynamics (VMD) [80] program was used to visualize the output.

\section{Orientation of the protein and inhibitor system for pulling simulations}

The DHP protein was rotated such that the pulling vector is generally in the $\mathrm{x}$-direction. It was assumed that the inhibitor would need to exit the distal pocket in the direction of the heme $\gamma$-edge, which is the shortest path for exit from the protein. This direction was defined by a vector from the center of mass of the protein to the 
center of mass of the inhibitor since it turns out that this vector is quite close to a vector from the $\mathrm{Fe}$ atom of the heme to the meso carbon on the $\gamma$-edge. These are atoms FE and $\mathrm{CMC}$, respectively in the coordinate file given in the Supporting Information. The vector along the x-direction was used to define pathway 2. A vector along the length of the inhibitor, i.e. a vector from the $\mathrm{Br}$ to the $\mathrm{O}$ atom of the inhibitor, 4-BP was used to define pathway 1 . The coordinates of the rotated protein aligned along the $\mathrm{x}$-axis are provided in the Supporting Information. The coordinates include the heme and 4-BP inhibitor and one $\mathrm{Na}$ atom required for charge neutrality. Using these coordinates the pulling vectors for pathways 1 and 2 were $0.786,-0.617$, 0.030 and 1.0, 0.0, 0.0, respectively. A vector orthogonal to pathway 1 and pointing along the $\delta$-edge of the heme was defined as pathway 3 . These vectors were used as pull_vec1 variable in in the GROMACS mdf file. The initial position of the 4-BP inhibitor (pull_init1) was $0.456,0.0,0.0$ in units of $\mathrm{nm}$.

\section{Parameters used for simulation of the inhibitor 4-BP}

The inhibitor studied in this work is 4-BP (designated as PBP in the Supporting Information coordinate and topology files). The parameters for 4-BP were obtained from a density functional theory (DFT) calculation of the molecule using $\mathrm{DMol}^{3}$.[81, 82] A geometry optimization and vibrational frequency calculation was conducted using the all-numerical basis set (DNP) and the PBE functional. [83] The geometry optimization was carried out until the convergence criterion of a change in energy of 
less than $10^{-6}$ Hartrees per iteration was achieved. The bond lengths and angles were obtained from the geometry optimized structure.

The Hessian matrix was constructed by finite difference using two displacements along each Cartesian coordinate of each atom to calculate the force of displacement. There were six zero frequencies indicative of the translational and rotational coordinates in the center-of-mass coordinate system. The remaining vibrational eigenvalues were positive. These eigenvalues were used to estimate the force constant matrix using the program FCARTP.[84] The values were also compared to the amino acid tyrosine to balance the force constants of the molecule. The bond stretching and angle bending force constants are given in the Supporting Information. The charges were obtained from Electrostatic Potential Fitting to the geometry optimized structure of 4-BP (see Supporting Information). [85]

\section{Steered molecular dynamics simulations using the Jarzynski equality}

By assumption, the minimum PMF pathway corresponds to the binding trajectory that has the lowest free energy barrier for exiting the protein. The minimum PMF is deemed closest to the actual binding free energy, since the lowest barrier for exiting the protein also should correspond to the lowest barrier for entry. Using established procedures the PMF was calculated from the pulling work using the Jarzynski equality: $[36,37]$

$$
e^{-\beta \Delta G}=\left\langle e^{-\beta W_{i}}\right\rangle
$$


where $\beta=1 / \mathrm{k}_{\mathrm{B}} \mathrm{T}$ and $\mathrm{W}_{\mathrm{i}}$ is the work of each pulling trajectory. Each PMF was calculated as the average of 100 trajectories of $20 \mathrm{~ns}$ each in order to approach reasonable sampling. Only an average of 10 trajectories of $20 \mathrm{~ns}$ were required for the the PMFs calculated for the fixed backbone. The simulation with no restraints resulted from an average of 200 simulations. The pathway comparison using SMD was made using a frozen protein backbone and then compared with US for validation.

The DHP + 4-BP system provides an opportunity to test the role of restraints. Since 4-BP has very limited degrees of freedom, because only the hydroxyl $\mathrm{H}$ atom has a torsional motion in 4-BP, no restraints are needed for the inhibitor. The protein backbone restraints were applied to each $\mathrm{C}, \mathrm{CA}$ and $\mathrm{N}$ atom in the sequence except the backbone atoms of residues K51 and P75 above the $\gamma$ edge. Those amino acids were not restrained to permit adjustments in the structure during the passage of the inhibitor through the protein. The pulling force constant has a significant effect on the stability of the less restrained SMD simulations. If the pulling force constant was set larger than $600 \mathrm{~kJ} /\left(\mathrm{mol} \mathrm{m^{2 }}\right)$ the simulations had large fluctuations in the PMF and sometimes failed completely as the restraint force constant was reduced. When pulling force constant was set to $2400 \mathrm{~kJ} /\left(\mathrm{mol} \mathrm{nm}^{2}\right)$ the simulations had a failure rate of $50 \%$ prior to the normal 20 ns endpoint. GROMACS is quite robust and the failure rate of pulling simulations is normally $<1 \%$. There were no failures observed for calculations using a frozen backbone so it was assumed that this problem is a 
consequence of the distortion of the protein under certain conditions where the pulling force constant exceeds the restraint force constant.

A study of the effect of the pulling force constant on the PMF was conducted for an unrestrained protein calculation. The values for the pulling force constant used were $300,600,1200,2400$ and $8000 \mathrm{~kJ} /\left(\mathrm{mol} \mathrm{nm}^{2}\right)$. Beyond the fact that there was an apparent instability for high values of the pulling force constant, no significant difference in the calculated PMF was found for different values of the pulling force constant less than $2400 \mathrm{~kJ} /\left(\mathrm{mol} \mathrm{m^{2 }}\right)$. The PMF was studied by SMD as a function of the restraint force constant from $5,10,200,500,1000 \mathrm{~kJ} /\left(\mathrm{mol} \mathrm{nm}{ }^{2}\right)$ using a pulling force constant of $1200 \mathrm{~kJ} /\left(\mathrm{mol} \mathrm{nm}{ }^{2}\right)$.

The inhibitor pulling velocity of $0.1 \mathrm{~nm} / \mathrm{ns}$ was determined based on a study of pulling velocity presented in the Supporting Information. Figure S3 shows that velocities of 0.2 and $0.5 \mathrm{~nm} / \mathrm{ns}$ provide a PMF that is significantly higher than 0.1 $\mathrm{nm} / \mathrm{ns}$. Figure S4 shows that there is no change in PMF as the pulling velocity is lowered from 0.1 to 0.05 and $0.02 \mathrm{~nm} / \mathrm{ns}$, which validates the use of a pulling velocity of $0.1 \mathrm{~nm} / \mathrm{ns}$ in the results presented below.

\section{Using non-equilibrium pulling simulations to determine the binding pathway}

One application of non-equilibrium pulling simulations is to determine the pathway used for entry and exit from the protein. It is difficult to experimentally verify such a determination other than to use the criterion that the minimum work 
done, i.e. minimum PMF is most likely to be the correct choice. Three pathways were defined for comparison. The direction of pathway 1 is along the vector from $\mathrm{Br}$ atom to the $\mathrm{O}$ atom in the hydroxyl group of the 4-BP molecule in the X-ray crystal structure (PDB 3LB2).[1] Figure 1 shows that the 4-BP trajectory in pathway 1 leads to exit through a gap between H55 and the $\gamma$-edge of the heme. Pathway 2 is parallel to the vector from the Fe atom to the $\gamma$-edge meso-carbon (Figure 1 and Figure S1). Residues K51 and H55 (marked in orange in Figure 1) are the main source of resistance in both of these exit pathways. Extensive experiments using diatomic ligands suggest that passage near H55 is the dominant pathway for water and diatomic ligands as they enter and exit the distal pocket. $[28,86]$ The above two choices for the pulling vector were contrasted with a third possible choice, which involves exit through the $\delta$-edge of the heme (Figure 1 and Figure S1). The $\delta$-edge of the heme has been implicated as the binding site in a number of peroxidases, which are related to DHP in terms of function, but not in terms of structure.

\section{Free energy calculation by Umbrella Sampling}

As a reference of comparison to the SMD results, the umbrella sampling with WHAM was also performed to calculate the free energy profile for the DHP-inhibitor separation using a restraint force constant of $1000 \mathrm{~kJ} /\left(\mathrm{mol} \mathrm{nm}{ }^{2}\right)$. The PMF was calculated along the pathway 1 . The total path with a length of $2 \mathrm{~nm}$ was divided into $0.1 \mathrm{~nm}$ wide equidistant windows. There were no additional restraints on the 4-BP molecule in $y$ and $z$-axis directions. The convergence within each window was 
checked (Figure S5) and overlap of the windows was also confirmed (Figure S6). [46] The corresponding validations of convergence and overlap for the frozen backbone method are given in Figures S7 and S8, respectively. To enhance sampling, the biased simulations were performed in 20 sampling windows, in which the center of mass of 4-BP was restrained along the pathway with a harmonic force constant of $600 \mathrm{~kJ} /(\mathrm{mol}$ $\mathrm{nm}^{2}$ ). As the same with the non-equilibrium method, the backbone atoms of the DHP molecule were harmonically restrained with a force constant of $1000 \mathrm{~kJ} /\left(\mathrm{mol} \mathrm{nm}{ }^{2}\right)$ in $x, y$ and $z$-axis (with the exception of the residues from K51 to P75 as discussed above for the SMD simulation). In each umbrella simulation window, a MD simulation of 14 ns was performed for WHAM procedure, in which the method of Bayesian bootstrapping of complete [35] is carried out to estimate the statistical uncertainty of the PMF profile. The biased ligand distributions obtained from the production runs are then unbiased and combined using WHAM to obtain the PMF of the ligand by the following equation (Eqn. 2):

$$
P M F(\zeta)=-R T \ln (p(\zeta))
$$

\section{Visualizing Pathways using Random Accelerated MD simulations}

Random Accelerated Molecular Dynamics (RAMD) studies were conducted in order to validate the possible molecular exit pathways from DHP found using the SMD method. [87] The RAMD method recently developed by some of the authors of this manuscript (L.L. and Q.W.) is derived from related methods that have been 
previously discussed. [88, 89] In RAMD a constant force with a random direction given by the vector $\vec{a}$ is applied to the target molecule at the start of the simulation. Using the definition of the displacement of the center of mass of a target molecule $\vec{d}$, the method is based on a comparison of the dot product $r=\vec{d} \cdot \vec{a}$ with a predefined threshold $r_{\text {min }}$, which is $0.02 \mathrm{~nm}$ in this work. The value of $\mathrm{r}$ represents the distance that should be covered by the target molecule in the direction of force during the time interval. At the end of each time interval $(0.25 \mathrm{ps})$ the evaluation is made and if $\mathrm{r}>$ $r_{\min }$ the force direction will not change in the next time interval. Otherwise, a new random force direction will be applied to the target molecule and the process will be repeated. The constant force should be carefully chosen to make sure the egress process occurs smoothly. A value of $200 \mathrm{~kJ} \mathrm{~mol}^{-1} \mathrm{~nm}^{-1}$ was found to be appropriate for the DHP + 4-BP complex. The RAMD simulation automatically stops either when the target molecule has translated $3 \mathrm{~nm}$ from its initial position or when the time interval number exceeds 4000.

\section{Results}

\section{SMD simulations along three pathways analyzed by the Jarzynski equality}

In order to obtain the lowest energy pathway for inhibitor exit from the distal pocket, we compared the calculated PMF using the Jarzynski equality along three pathways. A series of SMD simulations were performed along each of the three pulling vectors shown in Figure 1 and Figure S1 using a frozen backbone model. Only the $\gamma$ and $\delta$ 
heme edges have openings that are conceivable routes that would permit the inhibitor to pass (see Figure S1 for heme nomenclature). Two possible exit pathways for 4-BP through the $\gamma$ edge were found in a preliminary study using a partially frozen backbone (see Figure S2). A third pathway via the $\delta$ edge was considered to be of possible physiological relevance and was added for completeness. However, the pulling simulation along pathway 3 (directed to pass over the $\delta$ edge of the heme) was observed to result in exit of 4-BP through the $\gamma$ edge instead. The calculated PMF in that case was significantly higher than for the other two pathways (Figure S8), but this energy reflects the deviation of the inhibitor from intended pathway rather than an estimate of the barrier for passage over the $\delta$ edge. It was concluded that transit of the inhibitor along a pathway over the $\delta$ edge must be significantly higher in energy than those in the focus on a detailed comparison of pathways 1 and 2. The calculated PMFs shown in Figure S2 of the Supporting information indicate that pathway 1 has the lowest barrier for ligand exit. Therefore pathway 1 received further detailed study, because it is considered to be the best model for the physiologically relevant pathway found by the current methods.

Figure 2 shows the PMFs as a function of pulling distance along pathways 1 and 2. The different trajectories for entry and exit of 4-BP to and from the distal pocket are presented in Figure 3. The free energy differences are $107 \pm 8 \mathrm{~kJ} / \mathrm{mol}$ and $171 \pm 15$ $\mathrm{kJ} / \mathrm{mol}$ corresponding to pathway 1 and pathway 2 respectively. The standard deviation reported for pathway 2 corresponds to the statistical deviation of the PMF 
within a set of 10 averaged calculations. However, since pathway 1 had the lowest energy pathway found in these comparisons this pulling vector was selected for a full comparison of pulling speed, pulling force constant and restraint force constant. In pathway 1, the 4-BP molecule leaves the pocket through a gap between H55 and the heme molecule without significantly perturbing the protein. Thus, the free energy profile of pathway 1 indicates a relatively low barrier for the exit of 4-BP.

Regardless of the pulling vector, we find that the inhibitor must exit through an opening below H55 such that the inhibitor lies flat on the heme. If the vector is suboptimal the energy is much higher since the inhibitor crashes into amino acids of the distal pocket as it gropes its way towards the opening shown in Figure 3. For example, the significant increase in the free energy along pathway 2 results from the dislocation of residues $\mathrm{H} 55$ and $\mathrm{K} 51$ by the passage of 4-BP along this pathway. The increase in energy and distortion is even more extreme along pathway 3, but even for that pulling vector, which is orthogonal to pathway 1, the exit of 4-BP is still observed through the same opening beneath H55 and along the surface of the heme. Since the PMF of pathway 1 is the most direct pathway to this exit portal it is deemed to be the best model for inhibitor exit. Therefore, pathway 1 was further studied by US to validate the results of the calculation using the Jarzynski equality.

\section{Validation using umbrella sampling}


In order to establish a reference point for comparison with the non-equilibrium methods used in this study, the binding free energy of the DHP-inhibitor complex was calculated via US using equilibrium MD trajectories along trajectory 1. Figure 4 shows the PMF of the DHP-inhibitor separation process by umbrella sampling. The PMF obtained from US suggests a total free energy difference of $83 \pm 3 \mathrm{~kJ} / \mathrm{mol}$, which is significantly lower than the PMF calculated using SMD simulations with a frozen backbone $(107 \pm 5 \mathrm{~kJ} / \mathrm{mol})$. The overlaps for the various sampling windows and their convergence is presented in the Supporting Information. Figures S4 and S5 show the values for the frozen backbone and Figures S6 and S7 show the values for the calculation with a restraint force constant of $\left.1000 \mathrm{~kJ} /(\mathrm{mol} \mathrm{nm})^{2}\right)$. These are not significantly different, which is also consistent with the SMD results shown in Figure 5. We attempted to reduce the restraints for the US simulation as was done for the SMD. However, the individual window distribution functions became very broad when the restraints were reduced to $600 \mathrm{~kJ} /\left(\mathrm{mol} \mathrm{nm}{ }^{2}\right)$.

Intuitively, it makes sense that US will work well for calculation of the binding free energy as long as the binding molecule is restrained to be close to the target value for the windows of the umbrella simulation. In US the biasing force constant causes the inhibitor to fluctuate about a structure comprising a fixed distance between the protein and ligand. The distance must be well-defined, which means that the fluctuations of the inhibitor cannot be too large. If the biasing force constant is significantly below $1000 \mathrm{~kJ} /\left(\mathrm{mol} \mathrm{nm}{ }^{2}\right)$ the distribution of structures becomes so large 
that the WHAM analysis becomes impossible to execute. A systematic comparison of US as a function of pulling force constant fails for this reason. Realizing this we did not further pursue the reduction of the force constants using US and instead turned to a systematic comparison of the PMF calculated using the Jarzynski equality for various values of the restraint force constants.

\section{Validation using Random Accelerated Molecular Dynamic (RAMD) simulations}

The calculated pathways obtained by the RAMD technique are in good agreement with those obtained using SMD using a limited sampling of possible pathways. The exit pathways in the RAMD simulation are consistently observed to be in a relatively restricted region of the heme $\gamma$ edge. This path in the direction of the $\gamma$ edge presents the shortest distance for the 4-BP ligand to escape from the binding pocket of DHP (Figure 1c and Figure 1d). The results of the RAMD simulation are shown in Figures S13 and S14 of the Supporting Information. The two calculations correspond to 5 trajectories in a system with restraints of $1000 \mathrm{~kJ} /\left(\mathrm{mol} \mathrm{nm}{ }^{2}\right)$ and 50

$\mathrm{kJ} /\left(\mathrm{mol} \mathrm{nm}{ }^{2}\right)$, respectively. The trajectories in the more rigid system (Figure S13) all resemble the trajectories determined using SMD. There is greater variability in the system with weak constraints (Figure S14). Two of the five trajectories show no exit at all. Another two of the five exit through the heme $\gamma$ edge, as observed in the SMD simulations. One simulation shows another exit pathway, however, with a barrier that is significantly higher in energy than the principal one identified by SMD. 


\section{Calculation of the PMF as function of the pulling speed}

The SMD should be stable for pulling speeds that are sufficiently slow, so that the molecule can remain in equilibrium during the course of the simulation. Obviously, if the pulling speed is too great there will be irreversible changes in the system and will introduce artifacts into the simulation. hus, there is an optimum speed, which is the most rapid pulling spend possible that permits the system to remain in equilibrium. The systematic tests shown in Figures S2 and S3 reveal that the pulling speed of 0.1 $\mathrm{nm} / \mathrm{ns}$ is the optimum for the SMD simulations. Thus, the simulation time used in these studies is $20 \mathrm{~ns}$ per simulation given that the pulling distance for the inhibitor was $2 \mathrm{~nm}$ from the initial position inside the protein.

\section{Calculation of the PMF as function of the pulling force constant}

The first approximation was a frozen backbone simulation as discussed above, which was used to determine the lowest energy pathway for exit of the inhibitor. A pulling force constant of $8000 \mathrm{~kJ} /\left(\mathrm{mol} \mathrm{nm}{ }^{2}\right)$ was used for these simulations. Although this pulling force constant worked well for the frozen backbone simulation, it did not work particularly well for lower values of the restraint force constant. While the magnitude of the pulling force constant did not have a large effect on the calculated value of the PMF does affect the stability of the trajectory. After trials using 8000 ,

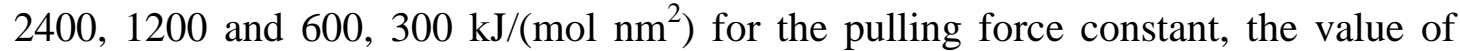


$1200 \mathrm{~kJ} /\left(\mathrm{mol} \mathrm{nm}{ }^{2}\right)$ was finally chosen for the systematic study of restraint force constants described in the next section.

\section{Calculation of the PMF as function of the protein backbone restraint}

Although restraints are often applied to pulling simulations, they present an artificial restriction that may result in systematic error in the calculated PMF. In order to systematically test the effect of restraints, the pulling simulations shown in Figure 5 were carried out with restraints backbone restraints in the series 1000, 500, 200, 10, 5 and $0 \mathrm{~kJ} /\left(\mathrm{mol} \mathrm{nm} \mathrm{nm}^{2}\right)$. The PMFs shown in Figure 5 are an average of 100 trajectories. For the pulling simulation with no constraints 200 trajectories were averaged, because of the much larger variety of DHP confirmations sampled in the absence of restratint.

Figure 5 shows that there is a systematic reduction in PMF calculated using the Jarzynski equality as the restraint force constant $\left(\mathrm{k}_{\mathrm{r}}\right)$ is decreased. It is obvious that run-to-run variability will increase as the restraint force constant is decreased. For the

series $1000,500,200,10,5$ and $0 \mathrm{~kJ} /\left(\mathrm{mol} \mathrm{nm}{ }^{2}\right)$ the calculated values of the PMF (with standard deviation in parentheses) were 103(8.4), 91(10.6), 87(12.6), 85(10.9), $79(27.0)$ and $53(25.0) \mathrm{kJ} / \mathrm{mol}$. Note that the standard deviation was approximately 40 $\mathrm{kJ} / \mathrm{mol}$ for the unrestrained simulation if only 100 averages were used. Thus, in general the standard deviation decreases approximately as $1 / \sqrt{N}$ where $\mathrm{N}$ is the number of averages. 
There is a disparity between the apparent convergence of the trend of decreasing restraints and the simulation with no restraints. If calculation without restraints is not considered it would appear that there is a trend towards a value of ca. $80 \mathrm{~kJ} / \mathrm{mol}$ for the PMF as $k_{r} \rightarrow 0$. However, the PMF of the simulation without restraints is 53 $\mathrm{kJ} / \mathrm{mol}$, which clearly does not agree with the trend. The origin of this discrepancy is not known at the present time. A universal issue for MD simulations is the possibility that greater sampling is needed to obtain robust average values.

\section{Discussion}

There are three significant findings in the present study. First, the results collectively show that the SMD approach can provide a method for the calculation of the pathways for ligand binding. Second, we find that it is possible to estimate the effect of restraints on the PMF by a systematic study of the energetic effect of the restraint force constant. Third, we find the reasonable agreement between simulation and experiment can obtained.

\section{Use of SMD to determine pathways in DHP}

There have been numerous proposals for increasing the utility of SMD for application of the calculation of the free energy of binding. Yamashita and Fujitani recognized the complexity of binding trajectories in the lysozyme-antibody binding process and recommended the use of multi-step targeted MD instead of SMD. [90] This approach is combined with biasing constraints [5, 17, 40, 42, 91-93] or random 
expulsion potentials $[7,8]$ in order to provide an appropriate sampling of the exit pathways. Fortunately, DHP does not have an extremely long tunnel for exit, which facilitated the comparison.

The RAMD simulations (Figures S13 and S14) validate the conclusions of the SMD in that the inhibitor (4-BP) exit is overwhelmingly observed to occur in the direction of the heme $\gamma$ edge as found by SMD. The simulations also give a molecular picture of the origin of the energetic factors that govern the escape barrier. There is a hydrogen bond between 4-BP and the heme propionate so that the inhibitor (4-BP) must overcome this bond energy and then surmount the steric blocking effect of the three residues H55, K51 or Y38 (represented by purple, green and yellow color in Figure 1a respectively) in order to escape. The RAMD calculations shown in Figures S13 and S14 show that the other directions $(\alpha, \beta$ and $\delta$ edge of the heme molecule) are all blocked consistent with the SMD results.

\section{Use of SMD to systematically test the effect of restraint force constants}

The results suggest that SMD is an appropriate method for systematic study of the effects of restraint force constants on the calculated PMF. As a corollary to the study of the effect of the restraint force constant we have obtained a simulation of the fully unrestrained PMF using 200 trajectories averaged trajectories. Even this number of trajectories may not provide sufficient sampling to obtain convergence for the unrestrained system, but the value obtained is reasonable and shows the potential for 
such calculations (see Figure 5 and Figures S9-S11 for the standard deviations for these calculations).

There is a significant difference between the calculated PMF in the limit as the restraint force constant, $\mathrm{k}_{\mathrm{r}} \rightarrow 0$ and in the unrestrained calculation. This suggests that additional sources of error must be considered. Protein rotation and drift can occur in the unrestrained simulation, which may introduce artifacts in the calculated PMF. As with any approach using $\mathrm{MD}$, sufficient sampling can provide an appropriate thermodynamic average. On the other hand, there are also possible artifacts in simulations that have restraints since these simulation may artificially restrict motion and thereby skew the sampled conformational distribution. Regardless of these potential problems the best agreement with experiment was obtained for the unrestrained simulation.

\section{The appropriateness of DHP(4-BP) binding for systematic SMD studies}

Clearly, the applicability of the SMD approach is system dependent. If either the protein or ligand has a large number of internal degrees of freedom, it is possible that the pulling force used in SMD could interfere with these motions and cause artifacts that decrease the accuracy of the free energy calculation. [15-17, 46] The interference is particularly large when there is a large electrostatic component to the binding free energy. [94-97] However, it may also be a problem when both protein and ligand have significant internal degrees of freedom. For these reasons we have focused on a 
relatively simple system as a test case for the values of the various parameters that enter into a non-equilibrium simulation. The only torsional degree of freedom in the inhibitor 4-BP involves the hydroxyl group. There is no significant electrostatic component to the binding of the inhibitor, 4-BP, since its $\mathrm{pKa}=9.3$, which means that it is essentially protonated at $\mathrm{pH}=7.0$, which is used in all experimental binding assays. Thus, we have used the protonated neutral form of 4-BP in the simulation. The properties of the inhibitor contrast with the substrates, 2,4,6-trichloro- and 2,4,6-tribromophenol, which are $\sim 75$ and $85 \%$ negatively charged $\mathrm{pH}=7.0$. [98-100] Since the inhibitor is not charged, solvation is not a major issue in the calculation. However, the system is fundamentally interesting because of the discovery of multiple functions in this relatively simple protein. $[51,101,102]$ It is also a valuable test case since there are crystal structures of six different molecules bound in the distal pocket and there clearly must be others that bind making this system a valuable one for future systematic comparisons of experimental and computation. [1, 27, 52, 53]

\section{Analysis of the experimental inhibitor binding affinity measurement}

The experimental determination of the binding constant is an important criterion for testing the accuracy of the results. To extract the experimental binding affinity, one must correct for the displacement of the $\mathrm{H}_{2} \mathrm{O}$ molecule from the heme Fe atom as shown in Scheme I. Unlike the $\mathrm{H}_{2} \mathrm{O}$ molecule, 4-BP has no covalent interaction with the heme Fe. The displacement of the $\mathrm{H}_{2} \mathrm{O}$ molecule indicated in Scheme 1 from the heme Fe has been observed both by X-ray crystallography and indirectly resonance 
Raman spectroscopy.[1] Thus, the experimental binding affinity $\Delta \mathrm{G}(\exp$.) includes the interactions of the 4-BP inhibitor $\Delta \mathrm{G}(4-\mathrm{BP})$, which are represented by the Jarzynski equality, but also the $\mathrm{H}_{2} \mathrm{O}$ molecules bonded to the heme Fe atom $\Delta \mathrm{G}\left(\mathrm{H}_{2} \mathrm{O}\right)$, which is not captured in the simulation conducted here. In fact, the energy required to break the $\mathrm{Fe}-\mathrm{OH}_{2}$ bond requires a quantum chemical calculation. Here, we have relied on the value calculated previously using B3LYP methods and validated by comparison with CCSD. [103] Thus, the MD simulation binding affinity must be corrected by including the $\mathrm{H}_{2} \mathrm{O}$ displacement energy in order to compare to the experimentally measured binding energies. (see Eqns. 3 and 4).

$$
\begin{aligned}
& \Delta \mathrm{G}(\text { exp. })=\Delta \mathrm{G}(4-\mathrm{BP})-\Delta \mathrm{G}\left(\mathrm{H}_{2} \mathrm{O}\right) \\
& \Delta \mathrm{G}(4-\mathrm{BP})=\Delta \mathrm{G}(\text { exp. })+\Delta \mathrm{G}\left(\mathrm{H}_{2} \mathrm{O}\right)
\end{aligned}
$$

In this equation $\Delta \mathrm{G}(4-\mathrm{BP})=\Delta \mathrm{G}_{\text {bind }}$, where $\Delta \mathrm{G}(4-\mathrm{BP})$ is the experimental quantity and $\Delta \mathrm{G}_{\text {bind }}$ is obtained from the SMD calculation. The experimental binding constant, $\mathrm{K}_{4-\mathrm{BP}}$, was measured by titration of 4-BP into DHP A using resonance Raman spectroscopy as the observable to determine of the extent of binding. The measurement was based on measurement of the $v_{3}$ Raman peak as a probe of five- and six-coordinate heme provides an estimate of $1.15 \mathrm{mM}$ for the binding constant of 4-BP in the distal pocket. [1, 104] This estimate is based on the assumption that 4-BP completely displaces the water molecule bound to the heme Fe atom when it enters the distal pocket, as observed in the X-ray crystal structure. [1] 
The value of $\Delta \mathrm{G}\left(\mathrm{H}_{2} \mathrm{O}\right)=-17 \mathrm{~kJ} / \mathrm{mol}$. We have recently estimated the value of the binding from inhibition kinetics using the Michaelis-Menten equation as well, which gives a binding free energy of $\Delta \mathrm{G}\left(\mathrm{H}_{2} \mathrm{O}\right)=-22 \mathrm{~kJ} / \mathrm{mol}$ at $298 \mathrm{~K}$. [52] The difference in these values is due to the difference in Fe ligation under the conditions of active turnover. Density functional theory (DFT) calculations give a value of $-53 \mathrm{~kJ} / \mathrm{mol}$ for the binding of $\mathrm{H}_{2} \mathrm{O}$. [103] Thus, using the experimental value for $298 \mathrm{~K}$, the actual value of the binding free energy of 4-BP should be $-75 \mathrm{~kJ} / \mathrm{mol}$ if all of the Fe atoms are bound to $\mathrm{H}_{2} \mathrm{O}$ initially. Both $\mathrm{X}$-ray crystallography and resonance Raman spectroscopy show that the fraction bound is $60 \%[1,104]$ and thus the expected energy should be $\sim-54 \mathrm{~kJ} / \mathrm{mol}$. The agreement is sufficiently good to justify further exploration of unrestrained simulations as a means to further develop applications of the Jarzynski equality for calculation of equilibrium binding of ligands.

\section{The significance of restraints in umbrella sampling}

Umbrella sampling (US) has become a very popular method for calculation of binding free energies. [93] US is implemented as a number of simulations in windows at defined distances along the pulling pathway. In each window a biasing force constant tethers the inhibitor to keep it within the range of distances defined by the window according to a biasing potential. In the simulations carried out on the DHP(4-BP) binding pair 20 windows were used, which were spaced $1 \mathrm{~nm}$ apart (see Figures S5 and S7). An idealized view of the Gaussian distribution for this set of windows is shown in Figure S11A. The Gaussian nature of the distribution results 
naturally from the quadratic form of the biasing potential and a theoretically Gaussian form for the histogram of conformations in each window (see Figure S11). [105]

The choice of the 20 windows in the US simulation was based on 20 target structures obtained from the SMD trajectory. Structures were chosen for defined distances between centers of mass of 4-BP and DHP from 0.0 to $2.0 \mathrm{~nm}$ in increments of $0.1 \mathrm{~nm}$. Of course, the success of US depends on an accurate determination of the pathway for binding, which must be obtained prior to the implementation. If we decrease either the biasing force constant (between DHP and 4-BP) or the restraint force constant (for the DHP backbone) there will be a greater distribution of conformations during the umbrella simulation due to the less stringent biasing potential. In order to carry out the WHAM procedure for averaging there must be appropriate overlap of DISTRIBUTIONS. [105-107] IF THE overlap is too small then there is no continuity, but if the distribution is too broad then the method loses resolution to distinguish regions of the trajectory. [105] Reducing the biasing force constant to values below $1000 \mathrm{~kJ} /\left(\mathrm{mol} \mathrm{nm}{ }^{2}\right)$ resulted in significant broadening of the distribution, which was not considered useful for further study.

The number of windows along the pulling trajectory must be sufficiently large to map out the change in mean force if one is to obtain a meaningful PMF from an US simulation. Since the breadth of the sampled conformational distribution depends on both the biasing and the restraint force constants, Figure S11 shows one cannot systematically decrease the force constants of the sampled distribution in the same 
way that we have done for the SMD simulation without broadening the distributions to the point where the US simulation becomes meaningless. For this reason one cannot conduct the control calculation in which the restraints are systematically lowered since the fluctuations become so large that the simulation no longer converges.

\section{The significance of restraints in steered molecular dynamics}

Based on the preceding considerations it appears that SMD presents the possibility of systematic study of the effect of pulling and restraint force constant. As Ytreberg has pointed out the effect of the restraint force constant on the free energy can be calculated using two correction terms. [50]

$\Delta G_{\text {bind }}=-W\left(r_{p}\right)+\Delta G_{\text {holo }}^{a}-k_{B} T \ln \left[\frac{r_{p}^{2}}{V_{0}} \int_{a p o} \cos \theta d \theta d \phi e^{-\beta U_{a}} \int_{\text {holo }} d r e^{-\beta W(r)}\right]$

The work term $-W\left(r_{p}\right)$ is calculated as the potential of mean force (PMF). The first correction term, $\Delta G_{\text {holo }}^{a}$, can be obtained from equilibrium methods, in which the potential energy of the protein is obtained as the restraint force constant is systematically increased in the same manner as the parameter $\lambda$ is increased from 0 to 1 in free energy perturbation calculations. The second term consists of a product of the apo integral, which can be calculated analytically using the geometry of the system weighted by the equilibrium energy of the protein and ligand as a function of the pulling trajectory. When there are no restraints, the correction terms are zero and $\Delta G_{\text {bind }}=-W\left(r_{p}\right)$, which means that $\Delta G_{\text {bind }}=-P M F$. [50] Thus, a restraint-free 
simulation can be directly compared to experiment. Using the approach of systematically lowering the magnitude of the restraints to estimate the magnitude of their contribution we find that the contribution of the restraints is $\sim 50 \mathrm{~kJ} / \mathrm{mol}$. This value is nearly equal to the absolute magnitude of the experimentally measured binding free energy. We find that corrections for the restraints are significant even for small molecule binding equilibria, in accord with the conclusion that has been reached for the more complex case of protein-protein binding equilibria. [21, 22, 44, 50]

\section{Conclusion}

We have addressed methods that can be used to calculate the free energy of binding of inhibitors and substrates in DHP. The Jarzynski equality has been employed to determine the pathway for inhibitor exit from the protein to calculate the binding affinity of the DHP-inhibitor complex. We have considered three possible pathways for inhibitor escape. These pathways are limited by the geometry of the inhibitor binding pocket, which is completely surrounded by the protein and blocked from exit by the protein leaving only the $\gamma$ and $\delta$ edge of the heme as possible directions for exit. The results calculated by the Jarzynski equality clearly show that pathway 1 over the $\gamma$ edge of the heme is the most likely pathway. The converged unrestrained Jarzynski equality for pathway 1 gives a dissociation energy of $\Delta \mathrm{G}=$ $53 \pm 25 \mathrm{~kJ} / \mathrm{mol}$. This value is in agreement with the binding energy of $54 \mathrm{~kJ} / \mathrm{mol}$ obtained from a combination of experimental measurement of 4-BP binding and DFT calculation of the $\mathrm{Fe}-\mathrm{H}_{2} \mathrm{O}$ binding energy. 
A systematic study of the effect of restraints has been conducted in order to understand the relationship of the SMD simulations with both experiment and with US simulations. Our study suggests that restraints can significantly increase the calculated free energy of binding, leading to poor agreement with experiment. These results suggest that the Jarzynski equality offers a method for the determination of binding pathways and binding energies by direct calculation of the unrestrained PMF.

\section{Acknowledgements}

This work was supported by the National Natural Science Foundation of China ((Grant Nos. 21074115, 21273200), MOE(J20091551,20090101110013) and Zhejiang University(2011XZZX002, 2011QNA3014)). SF Acknowledges support by the Guangbiao Scholars program and ARO grant 56781-LS. We acknowledge the use of the ARC computing resource at NC State University, which was supported by NSF grant 0958311.

\section{References}

[1] M.K.D. Thompson, M.F.; de Serrano V.; Nicoletti, F.P.; Howes, B.D.; Smulevich, G.; Franzen, S. , Internal Binding of Halogenated Phenols in Dehaloperoxidase-Hemoglobin Inhibits Peroxidase Function., Biophys. J., 99 (2010) 1586-1595.

[2] S. Franzen, M.K. Thompson, R.A. Ghiladi, The Dehaloperoxidase Paradox, Biochim. Biophys. Acta, 1824 (2012) 578-588.

[3] W.L. Jorgensen, The many roles of computation in drug discovery, Science, 303 (2004) 1813-1818.

[4] P.A. Kollman, I. Massova, C. Reyes, B. Kuhn, S.H. Huo, L. Chong, M. Lee, T. Lee, Y. Duan, W. Wang, O. Donini, P. Cieplak, J. Srinivasan, D.A. Case, T.E. 
Cheatham, Calculating structures and free energies of complex molecules: Combining molecular mechanics and continuum models, Acc. Chem. Res., 33 (2000) 889-897.

[5] L.J. Kingsley, M.A. Lill, Including Ligand-Induced Protein Flexibility into Protein Tunnel Prediction, J. Comp. Chem., 35 (2014) 1748-1756.

[6] H. Fukunishi, J. Shimada, K. Shiraishi, Antigen-Antibody Interactions and Structural Flexibility of a Femtomolar-Affinity Antibody, Biochemistry, 51 (2012) 2597-2605.

[7] W. Li, J. Shen, G. Liu, Y. Tang, T. Hoshino, Exploring coumarin egress channels in human cytochrome P450 2A6 by random acceleration and steered molecular dynamics simulations, Prot.-Struct. Func. Bioinform., 79 (2011) 271-281.

[8] S.K. Ludemann, V. Lounnas, R.C. Wade, How do substrates enter and products exit the buried active site of cytochrome P450cam? 1. Random expulsion molecular dynamics investigation of ligand access channels and mechanisms, J. Mol. Biol., 303 (2000) 797-811.

[9] S.K. Ludemann, V. Lounnas, R.C. Wade, How do substrates enter and products exit the buried active site of cytochrome P450cam? 2. Steered molecular dynamics and adiabatic mapping of substrate pathways, J. Mol. Biol., 303 (2000) 813-830.

[10] F. Forti, L. Boechi, D.A. Estrin, M.A. Marti, Comparing and Combining Implicit Ligand Sampling with Multiple Steered Molecular Dynamics to Study Ligand Migration Processes in Heme Proteins, J. Comp. Chem. , 32 (2011) 2219-2231

1.

[11] R. Yu, Q. Kaas, D.J. Craik, Delineation of the Unbinding Pathway of alpha-Conotoxin Iml from the alpha 7 Nicotinic Acetylcholine Receptor, J. Phys. Chem. B, 116 (2012) 6097-6105.

[12] D.Q. Zhang, J. Gullingsrud, J.A. McCammon, Potentials of mean force for acetylcholine unbinding from the alpha7 nicotinic acetylcholine receptor ligand-binding domain, J. Am. Chem. Soc., 128 (2006) 3019-3026.

[13] Z. Shen, F. Cheng, Y. Xu, J. Fu, W. Xiao, J. Shen, G. Liu, W. Li, Y. Tang, Investigation of Indazole Unbinding Pathways in CYP2E1 by Molecular Dynamics Simulations, Plos One, 7 (2012).

[14] J.-L. Zhang, Q.-C. Zheng, Z.-Q. Li, H.-X. Zhang, Molecular Dynamics Simulations Suggest Ligand's Binding to Nicotinamidase/Pyrazinamidase, Plos One, 7 (2012) Art. No. e39546.

[15] T. Bastug, P.C. Chen, S.M. Patra, S. Kuyucak, Potential of mean force calculations of ligand binding to ion channels from Jarzynski's equality and umbrella sampling, J. Chem. Phys., 128 (2008) Art. No. t155104. 
[16] M.A. Cuendet, O. Michielin, Protein-protein interaction investigated by steered molecular dynamics: The TCR-pMHC complex, Biophys. J., 95 (2008) 3575-3590.

[17] E. Piccinini, M. Ceccarelli, F. Affinito, R. Brunetti, C. Jacoboni, Biased molecular simulations for free-energy mapping: A comparison on the KcsA channel as a test case, J. Chem. Theory Comput., 4 (2008) 173-183.

[18] L.Y. Chen, Hybrid Steered Molecular Dynamics Approach to Computing Absolute Binding Free Energy of Ligand-Protein Complexes: A Brute Force Approach That Is Fast and Accurate, J. Chem. Theo. Comp., 11 (2015) 1928-1938.

[19] R. Alhadeff, A. Ganoth, I.T. Arkin, Mechanistic studies of the apical sodium-dependent bile acid transporter, Prot.-Struct. Func. Bioinform., 83 (2015) 1107-1117.

[20] P. Nicolini, D. Frezzato, C. Gellini, M. Bizzarri, R. Chelli, Toward quantitative estimates of binding affinities for protein-ligand systems involving large inhibitor compounds: A steered molecular dynamics simulation route, J. Comp. Chem., 34 (2013) 1561-1576.

[21] J.C. Gumbart, B. Roux, C. Chipot, Standard Binding Free Energies from Computer Simulations: What Is the Best Strategy?, J. Chem. Theory Comp.,, 9 (2013) 794-802.

[22] J.C. Gumbart, B. Roux, C. Chipot, Efficient Determination of Protein-Protein Standard Binding Free Energies from First Principles, J. Comp. Chem., 9 (2013).

[23] S. Doudou, N.A. Burton, R.H. Henchman, Standard Free Energy of Binding from a One-Dimensional Potential of Mean Force, J. Chem. Theory Comp., 5 (2009) 909-918.

[24] T.W. Allen, O.S. Andersen, B. Roux, Energetics of ion conduction through the gramicidin channel, Proc. Natl. Acad. Sci. U.S.A., 101 (2004) 117-122.

[25] M.W. LaCount, Zhang, E. L., Chen, Y. P., Han, K. P., Whitton, M. M., Lincoln, D. E., Woodin, S. A., and Lebioda, L., The crystal structure and amino acid sequence of Dehaloperoxidase from Amphitrite ornata indicate common ancestry with globins, J. Biol. Chem., 275 (2000) 18712-18716.

[26] L. Lebioda, LaCount, M. W., Zhang, E., Chen, Y. P., Han, K., Whitton, M. M., Lincoln, D. E., and Woodin, S. A. , An enzymatic globin from a marine worm, Nature, 401 (1999) 445.

[27] V. de Serrano, Z.X. Chen, M.F. Davis, S. Franzen, X-ray crystal structural analysis of the binding site in the ferric and oxyferrous forms of the recombinant heme dehaloperoxidase cloned from Amphitrite ornata, Acta Cryst. D-Biol. Cryst., 63 (2007) 1094-1101. 
[28] J. Zhao, V. de Serrano, S. Franzen, A Model for the Flexibility of the Distal Histidine in Dehaloperoxidase-Hemoglobin A Based on X-ray Crystal Structures of the Carbon Monoxide Adduct, Biochemistry, 53 (2014) 2474-2482.

[29] M.K. Thompson, S. Franzen, R.A. Ghiladi, B.J. Reeder, D.A. Svistunenko, Compound ES of Dehaloperoxidase Decays via Two Alternative Pathways Depending on the Conformation of the Distal Histidine, J. Am. Chem. Soc., 132 (2010) 17501-17510.

[30] K.N. Nienhaus, E.; Davis, M. F.; Franzen, S.; Nienhaus, G. U., Determinants of Substrate Internalization in the Distal Pocket of Dehaloperoxidase Hemoglobin of Amphitrite ornata, Biochemistry, 47 (2008) 12985-12994.

[31] Z. Chen, de Serrano, V., Betts, L., Franzen, S. , Distal histidine conformation flexibility in dehaloperoxidase from Amphitrite ornata, Acta Cryst., D65 (2009) 34-40.

[32] M.F.B. Davis, B. G.; Franzen, S. , Determination of Separate Inhibitor and Substrate Binding Sites in the Dehaloperoxidase-Hemoglobin from Amphitrite ornata, Biochemistry, 49 (2010) 1199-1206.

[33] M.F. Davis, H. Gracz, F.A.P. Vendeix, V. de Serrano, A. Somasundaram, S.M. Decatur, S. Franzen, Different Modes of Binding of Mono-, Di-, and Trihalogenated Phenols to the Hemoglobin Dehaloperoxidase from Amphitrite ornata, Biochemistry, 48 (2009) 2164-2172.

[34] M.K. Gilson, H.-X. Zhou, Calculation of protein-ligand binding affinities, Ann. Rev. Biophys. Biomol. Struct.2007, pp. 21-42.

[35] J.S. Hub, B.L. de Groot, D. van der Spoel, g_wham-A Free Weighted Histogram Analysis Implementation Including Robust Error and Autocorrelation Estimates, J. Chem. Theory Comput., 6 (2010) 3713-3720.

[36] C. Jarzynski, Equilibrium free-energy differences from nonequilibrium measurements: A master-equation approach, Phys. Rev. E, 56 (1997) 5018-5035.

[37] C. Jarzynski, Nonequilibrium equality for free energy differences, Phys. Rev.Lett., 78 (1997) 2690-2693.

[38] D.A. Hendrix, C. Jarzynski, A "fast growth" method of computing free energy differences, J. Chem. Phys., 114 (2001) 5974-5981.

[39] R.C. Lua, A.Y. Grosberg, Practical applicability of the Jarzynski relation in statistical mechanics: A pedagogical example, J. Phys.Chem. B, 109 (2005) 6805-6811.

[40] J. Gore, F. Ritort, C. Bustamante, Bias and error in estimates of equilibrium free-energy differences from nonequilibrium measurements, Proc. Natl. Acad. Sci. U.S.A., 100 (2003) 12564-12569. 
[41] G. Hummer, Fast-growth thermodynamic integration: Error and efficiency analysis, J. Chem. Phys., 114 (2001) 7330-7337.

[42] H. Oberhofer, C. Dellago, P.L. Geissler, Biased sampling of nonequilibrium trajectories: Can fast switching simulations outperform conventional free energy calculation methods?, J. Phys. Chem. B, 109 (2005) 6902-6915.

[43] D. Rodriguez-Gomez, E. Darve, A. Pohorille, Assessing the efficiency of free energy calculation methods, J. Chem. Phys., 120 (2004) 3563-3578.

[44] F.M. Ytreberg, D.M. Zuckerman, Efficient use of nonequilibrium measurement to estimate free energy differences for molecular systems, J. Comp. Chem., 25 (2004) 1749-1759.

[45] C. Oostenbrink, W.F. van Gunsteren, Calculating zeros: Non-equilibrium free energy calculations, Chem. Phys., 323 (2006) 102-108.

[46] P.C. Chen, S. Kuyucak, Accurate Determination of the Binding Free Energy for KcsA-Charybdotoxin Complex from the Potential of Mean Force Calculations with Restraints, Biophys. J., 100 (2011) 2466-2474.

[47] D.S. Dashti, A.E. Roitberg, Optimization of Umbrella Sampling Replica Exchange Molecular Dynamics by Replica Positioning, Journal of Chemical Theory and Computation, 9 (2013) 4692-4699.

[48] B. Ilan, E. Tajkhorshid, K. Schulten, G.A. Voth, The mechanism of proton exclusion in aquaporin channels, Prot. Struct. Func. Bioinform., 55 (2004) 223-228.

[49] R. Amaro, E. Tajkhorshid, Z. Luthey-Schulten, Developing an energy landscape for the novel function of a (beta/alpha)(8) barrel: Ammonia conduction through HisF, Proc. Natl. Acad. Sci. U.S.A., 100 (2003) 7599-7604.

[50] F.M. Ytreberg, Absolute FKBP binding affinities obtained via nonequilibrium unbinding simulations, J. Chem. Phys., 130 (2009) Art. No. 164906.

[51] D.A. Barrios, J. D'Antonio, N.L. McCombs, J. Zhao, S. Franzen, A.C. Schmidt, L.A. Sombers, R.A. Ghiladi, Peroxygenase and Oxidase Activities of Dehaloperoxidase-Hemoglobin from Amphitrite ornata, J. Am. Chem. Soc., 136 (2014) 7914-7925.

[52] J. Zhao, V.S. de Serrano, J. Zhao, S. Franzen, Structural and kinetic study of an internal substrate binding site of dehaloperoxidase-hemoglobin A from Amphitrite ornata, Biochemistry, 52 (2013) 2427-2439.

[53] C. Wang, L.L. Lovelace, S. Sun, J.H. Dawson, L. Lebioda, Complexes of Dual-Function Hemoglobin/Dehaloperoxidase with Substrate 2,4,6-Trichlorophenol Are Inhibitory and Indicate Binding of Halophenol to Compound I, Biochemistry, 52 (2013) 6203-6210. 
[54] K. Schleinkofer, Sudarko, P.J. Winn, S.K. Ludemann, R.C. Wade, Do mammalian cytochrome P450s show multiple ligand access pathways and ligand channelling?, Embo Reports, 6 (2005) 584-589.

[55] J. Zhao, S. Franzen, Study of mechanism of the kinetic inhibition of dehaloperoxidase-hemoglobin, J. Phys. Chem. B, 117 (2013) 8301-8309.

[56] V.S. de Serrano, S. Franzen, Structural Evidence for Stabilization of Inhibitor Binding by a Protein Cavity in the Dehaloperoxidase-Hemoglobin from Amphitrite ornata, Peptide Sci., 98 (2012) 27-35.

[57] E. Chovancova, A. Pavelka, P. Benes, O. Strnad, J. Brezovsky, B. Kozlikova, A. Gora, V. Sustr, M. Klvana, P. Medek, L. Biedermannova, J. Sochor, J. Damborsky, CAVER 3.0: A Tool for the Analysis of Transport Pathways in Dynamic Protein Structures, Plos Comp. Biol., 8 (2012).

[58] M. Petrek, P. Kosinova, J. Koca, O. M, MOLE: A Voronoi diagrambased

explorer of molecular channels, pores, and tunnels, Structure, 15 (2007) 1357-1363.

[59] J. Zhao, M. Xue, D. Gudanis, H. Gracz, G. Findenegg, Z. Gdaniec, S. Franzen, Structure and Backbone Dynamics of Dehaloperoxidase-Hemoglobin A: Insights from NMR relaxation spectroscopy and Molecular Dynamics Simulations, (Manuscript in preparation).

[60] Y. Zhang, L. Liu, L. Wu, S. Li, F. Li, Z. Li, Theoretical investigation on the diatomic ligand migration process and ligand binding properties in non-O-2-binding H-NOX domain, Prot.-Struct. Func. Bioinform., 81 (2013) 1363-1376.

[61] J. Cohen, A. Arkhipov, R. Braun, K. Schulten, Imaging the migration pathways for O-2, CO, NO, and Xe inside myoglobin, Biophys. J., 91 (2006) 1844-1857.

[62] R. Elber, M. Karplus, Enhanced Sample in Molecular Dynamics - Use of the Time-Dependent Hartree Approximation for a Simulation of Carbon Monoxide Diffusion through Myoglobin, J. Am. Chem. Soc., 112 (1990) 9161-9175.

[63] J.E. Knapp, R. Pahl, J. Cohen, J.C. Nichols, K. Schulten, Q.H. Gibson, V. Srajer, W.E. Royer, Ligand Migration and Cavities within Scapharca Dimeric Hbl: Studies by Time-Resolved Crystallography, Xe Binding, and Computational Analysis, Structure, 17 (2009) 1494-1504.

[64] A.D. MacKerell, D. Bashford, M. Bellott, R.L. Dunbrack, J.D. Evanseck, M.J. Field, S. Fischer, J. Gao, H. Guo, S. Ha, D. Joseph-McCarthy, L. Kuchnir, K. Kuczera, F.T.K. Lau, C. Mattos, S. Michnick, T. Ngo, D.T. Nguyen, B. Prodhom, W.E. Reiher, B. Roux, M. Schlenkrich, J.C. Smith, R. Stote, J. Straub, M. Watanabe, J. Wiorkiewicz-Kuczera, D. Yin, M. Karplus, All-atom empirical potential for molecular modeling and dynamics studies of proteins, J. Phys. Chem. B, 102 (1998) 3586-3616. 
[65] R. Elber, Q.H. Gibson, Toward quantitative simulations of carbon monoxide escape pathways in myoglobin, J. Phys. Chem. B, 112 (2008) 6147-6154.

[66] Y. Nishihara, M. Sakakura, Y. Kimura, M. Terazima, The escape process of carbon monoxide from myoglobin to solution at physiological temperature, J. Am. Chem. Soc., 126 (2004) 11877-11888.

[67] J.Z. Ruscio, D. Kumar, M. Shukla, M.G. Prisant, T.M. Murali, A.V. Onufriev, Atomic level computational identification of ligand migration pathways between solvent and binding site in myoglobin, Proc. Natl. Acad. Sci. U.S.A., 105 (2008) 9204-9209.

[68] M.K. Thompson, S. Franzen, M.F. Davis, R.C. Oliver, J.K. Krueger, Dehaloperoxidase-Hemoglobin from Amphitrite ornata Is Primarily a Monomer in Solution, J. Phys. Chem. B, 115 (2011) 4266-4272.

[69] M. Nategholeslam, C.G. Gray, B. Tomberli, Implementation of the Forward-Reverse Method for Calculating the Potential of Mean Force Using a Dynamic Restraining Protocol, J. Phys. Chem. B, 118 (2014) 14203-14214.

[70] A. Dryga, A. Warshel, Renormalizing SMD: The Renormalization Approach and Its Use in Long Time Simulations and Accelerated PAU Calculations of Macromolecules, J. Phys. Chem. B, 114 (2010) 12720-12728.

[71] R. Chelli, Local Sampling in Steered Monte Carlo Simulations Decreases Dissipation and Enhances Free Energy Estimates via Nonequilibrium Work Theorems, J. Chem. Theo. Comp., 8 (2012) 4040-4052.

[72] H.J. Woo, B. Roux, Calculation of absolute protein-ligand binding free energy from computer simulations, Proc. Natl. Acad. Sci. U.S.A., 102 (2005) 6825-6830.

[73] K.L. Whalen, K.M. Chang, M.A. Spies, Hybrid Steered Molecular Dynamics-Docking: An Efficient Solution to the Problem of Ranking Inhibitor Affinities Against a Flexible Drug Target, Molec. Inform., 30 (2011) 459-471.

[74] D. Van der Spoel, E. Lindahl, B. Hess, G. Groenhof, A.E. Mark, H.J.C. Berendsen, GROMACS: Fast, flexible, and free, J. Comp. Chem., 26 (2005) 1701-1718.

[75] B. Hess, P-LINCS: A parallel linear constraint solver for molecular simulation, J. Chem. Theory Comp., 4 (2008) 116-122.

[76] W.L. Jorgensen, C. Jenson, Temperature dependence of TIP3P, SPC, and TIP4P water from NPT Monte Carlo simulations: Seeking temperatures of maximum density, J.f Comp. Chem., 19 (1998) 1179-1186.

[77] S. Miyamoto, P.A. Kollman, SETTLE - An Analytical Version of the SHAKE and RATTLE Algorithm for Rigid Water Models, J. Comp. Chem., 13 (1992) 952-962. 
[78] G. Bussi, D. Donadio, M. Parrinello, Canonical sampling through velocity rescaling, J. Chem. Phys., 126 (2007) No. 014101.

[79] T. Darden, D. York, L. Pedersen, Particle Mesh Ewald - An Nlog(N) Method for Eward Sums in Large Systems, J. Chem. Phys., 98 (1993) 10089-10092.

[80] W. Humphrey, A. Dalke, K. Schulten, VMD: Visual molecular dynamics, J. Mol. Graph., 14 (1996) 33-41.

[81] B. Delley, An All-Electron Numerical-Method for Solving the Local Density Functional for Polyatomic-Molecules, J. Chem. Phys., 92 (1990) 508-517.

[82] B. Delley, From molecules to solids with the DMol(3) approach, J. Chem. Phys., 113 (2000) 7756-7764.

[83] J.P. Perdew, K. Burke, M. Ernzerhof, Generalized gradient approximation made simple, Phys. Rev. Lett., 77 (1996) 3865-3868.

[84] W.B. Collier, T.D. Klots, Heteroatom Derivatives of Indene 1. Vibrational Frequencies and a Refined Scaled Overlay of the AM1 Force FIeld of Indole, Benzofuran, Benzothiophene, Benzoxazole and Benzothiazole, Spectrochim. Acta a-Mol. Biomol. Spec., 51 (1995) 1255-1272.

[85] C.I. Bayly, P. Cieplak, W.D. Cornell, P.A. Kollman, A Well-Behaved Electrostatic Potential Based Method Using Charge Restraints for Deriving Atomic Charges - The RESP Model, J. Phys. Chem., 97 (1993) 10269-10280.

[86] J.J. Zhao, V. de Serrano, R. Dumarieh, M. Thompson, R.A. Ghiladi, S. Franzen, The Role of the Distal Histidine in $\mathrm{H} 2 \mathrm{O} 2$ Activation and Heme Protection in both Peroxidase and Globin Functions, J. Phys. Chem. B, 116 (2012) 12065-12077.

[87] J. Zhang, D. Li, T. Sun, L. Liang, Q. Wang, Interaction of P-glycoprotein with anti-tumor drugs: the site, gate and pathway., Soft Matter, 11 (2015) 6633-6639.

[88] S.K. Ludemanna, V. Lounnasa, R.C. Wadea, How do Substrates Enter and Products Exit the Buried Active Site of Cytochrome P450cam? 1. Random Expulsion Molecular Dynamics Investigation of Ligand Access Channels and Mechanisms, J. Mol. Biol., 303 (2000) 797-811

[89] D. Long, Y. Mu, D. Yang, Molecular dynamics simulation of ligand dissociation from liver fatty acid binding protein, PLoS One, 4 (2009) e6081.

[90] T. Yamashita, H. Fujitani, On accurate calculation of the potential of mean force between antigen. and antibody: A case of the HyHEL-10-hen egg white lysozyme system, Chem. Phys. Lett., 609 (2014) 50-53.

[91] E. Darve, D. Rodriguez-Gomez, A. Pohorille, Adaptive biasing force method for scalar and vector free energy calculations, J. Chem. Phys., 128 (2008) Art. No. 144120. 
[92] J. Gumbart, Exploring substrate diffusion in channels using biased molecular dynamics simulations, Methods in molecular biology (Clifton, N.J.), 914 (2012) 337-350.

[93] E. Rosta, G. Hummer, Free Energies from Dynamic Weighted Histogram Analysis Using Unbiased Markov State Model, J. Chem. Theo. Comp., 11 (2015) 276-285.

[94] A.E. Cho, V. Guallar, B.J. Berne, R. Friesner, Importance of accurate charges in molecular docking: Quantum mechanical/molecular mechanical (QM/MM) approach, J. Comp. Chem., 26 (2005) 915-931.

[95] Y. Tong, Y. Mei, Y.L. Li, C.G. Ji, J.Z.H. Zhang, Electrostatic Polarization Makes a Substantial Contribution to the Free Energy of Avidin-Biotin Binding, J. Am. Chem. Soc., 132 (2010) 5137-5142.

[96] A. Warshel, P.K. Sharma, M. Kato, W.W. Parson, Modeling electrostatic effects in proteins, Biochim. Biophys. Acta Prot. Proteom., 1764 (2006) 1647-1676.

[97] D. Jiao, P.A. Golubkov, T.A. Darden, P. Ren, Calculation of protein-ligand binding free energy by using a polarizable potential, Proc. Natl. Acad. Sci. U.S.A., 105 (2008) 6290-6295.

[98] S. Franzen, L.B. Gilvey, J.L. Belyea, The $\mathrm{pH}$ dependence of the activity of dehaloperoxidase from Amphitrite ornata, Biochim. Biophys. Acta-Prot. Proteom., 1774 (2007) 121-130.

[99] G. Schkolnik, T. Utesch, J.J. Zhao, S. Jiang, M.K. Thompson, M.A. Mroginski, P. Hildebrandt, S. Franzen, Catalytic efficiency of dehaloperoxidase A is controlled by electrostatics - application of the vibrational Stark effect to understand enzyme kinetics, Biochem. BIophys. Res. Comm., 430 (2013) 1011-1015.

[100] J.J. Zhao, J. Rowe, J. Franzen, C. He, S. Franzen, Study of the electrostatic effects of mutations on the surface of dehaloperoxidase-hemoglobin A, Biochem. Biophys. Res. Comm., 420 (2012) 733-737.

[101] J. Belyea, Gilvey, L. B., Davis, M. F., Godek, M., Sit, T. L., Lommel, S. A., Franzen, S. , Enzyme Function of the Globin Dehaloperoxidase from Amphitrite ornate Is Activated by Substrate Binding, Biochemistry, 44 (2005) 15637-15644.

[102] S.A.W. Y. P. Chen, D.E. Lincoln, C.R. Lovell, An unusual dehalogenating peroxidase from the marine terebellid polychaete Amphitrite ornata, J. Biol. Chem., 271 (1996) 4609-4612.

[103] N. Strickland, J.N. Harvey, Spin-forbidden ligand binding to the ferrous-heme group: Ab initio and DFT studies, J. Phys. Chem. B, 111 (2007) 841-852.

[104] F.P. Nicoletti, Thompson, M. K., Howes, B. D., Franzen, S., Smulevich, G. , New Insights into the Role of Distal Histidine Flexibility in Ligand Stabilization 
of Dehaloperoxidase-Hemoglobin from Amphitrite ornata, Biochemistry, 49 (2010) $1903-1912$.

[105] R.I. Cukier, Variance of a Potential of Mean Force Obtained Using the Weighted Histogram Analysis Method, J. Phys. Chem. B, 117 (2013) 14785-14796.

[106] S. Kumar, D. Bouzida, R.H. Swendsen, P.A. Kollman, J.M. Rosenberg, The Weighted Histogram Analysis Method for Free Energy Calculations on Biomolecules. 1. The Method, J. Comp. Chem., 13 (1992) 1011-1021.

[107] A.M. Ferrenberg, R.H. Swendsen, Optimized Monte-Carlo Data Analysis, Phys. Rev. Lett., 63 (1989) 1195-1198. 


\section{Figures}

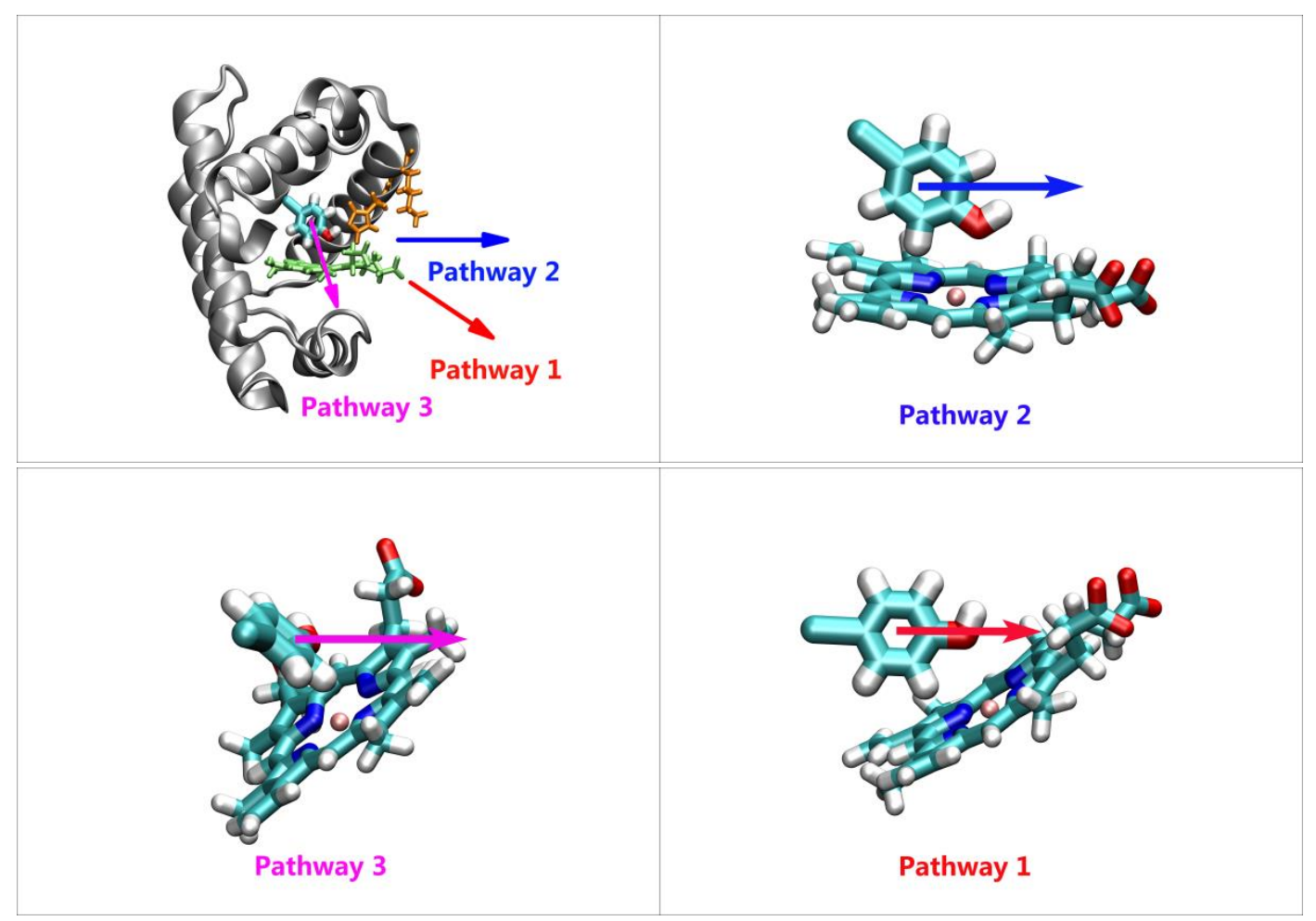

Figure 1. Schematic representation of the three pathways adopted in the SMD simulations for the DHP-4-BP complex. Pathway 1 is defined by the vector of the $\mathrm{Br}$

- $\mathrm{O}$ distance. This vector aimed downward towards $-\mathrm{y}$ and is $\sim 50^{\circ}$ from the $\mathrm{x}$-direction. Pathway 2 is along the $\mathrm{x}$-axis and bisects the two propionate groups on the $\gamma$-edge of the heme. Pathway 3 is directed towards the $\delta$-edge of the heme and is along the $\mathrm{z}$-axis and $-\mathrm{y}$-axis. 


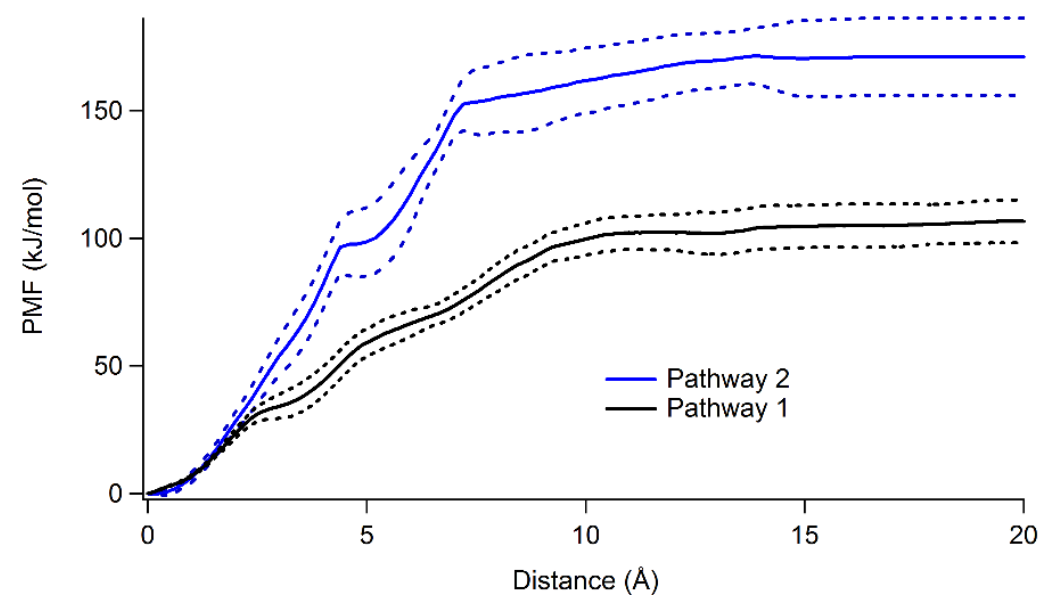

Figure 2. PMF for the protein-inhibitor separation as a function of pulling distance calculated by the Jarzynski equality along two pathways. These simulations were the result of 10 averaged trajectories carried out with a frozen backbone. The error bars are shown as dashed lines above and below the calculated PMF representing the standard deviations from the average of 100 trajectories. 


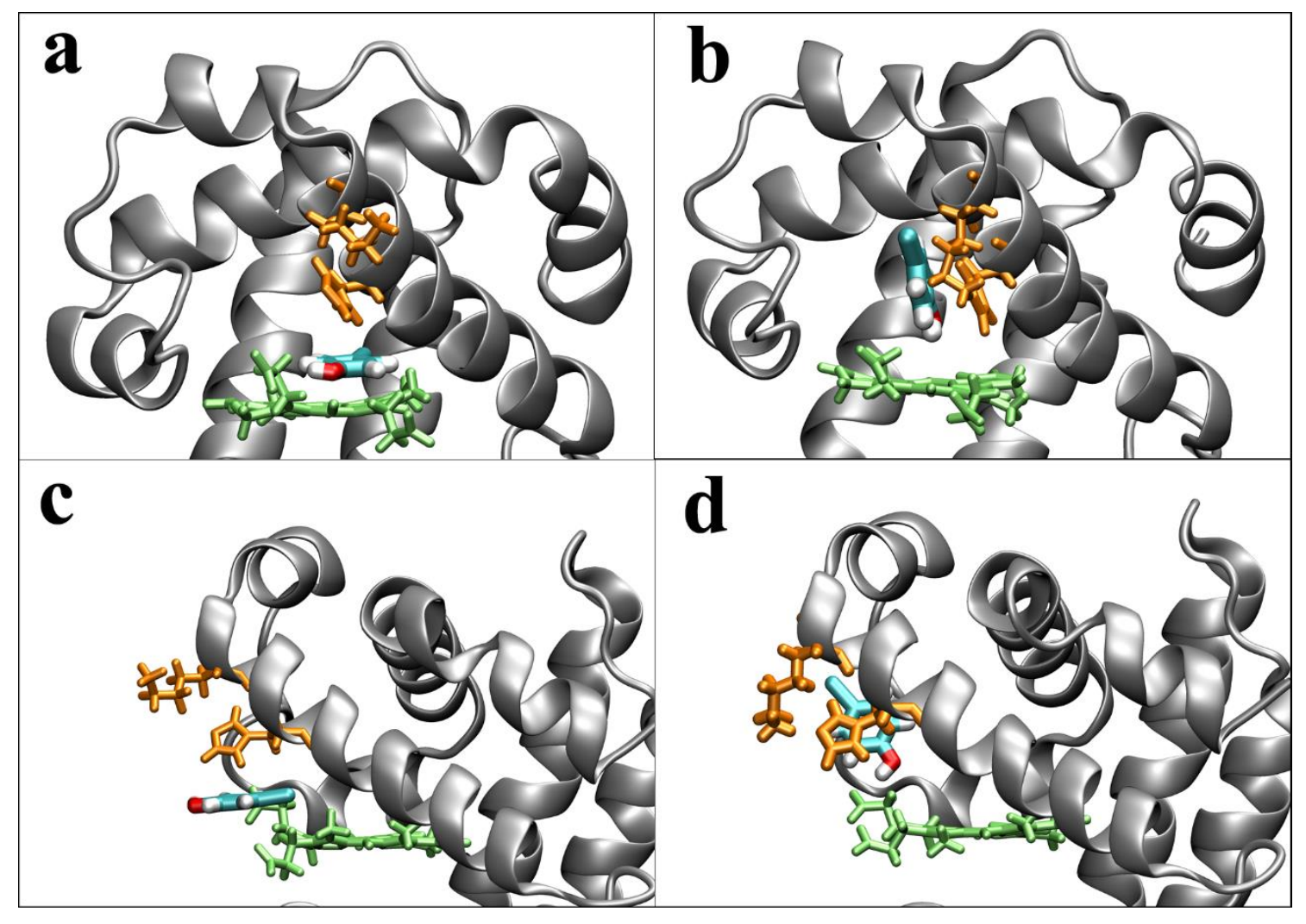

Figure 3. a) and c): Configurational snapshot of 4-BP molecule exiting from the binding site in the distal pocket of DHP in the SMD simulation for pathway 1. The 4-BP molecule leaves the pocket through a gap between H55 and the heme. b) and d): Similar snapshot of the SMD simulation for pathway 2. The 4-BP molecule pushes residues $\mathrm{K} 51$ and residue $\mathrm{H} 55$ away. 


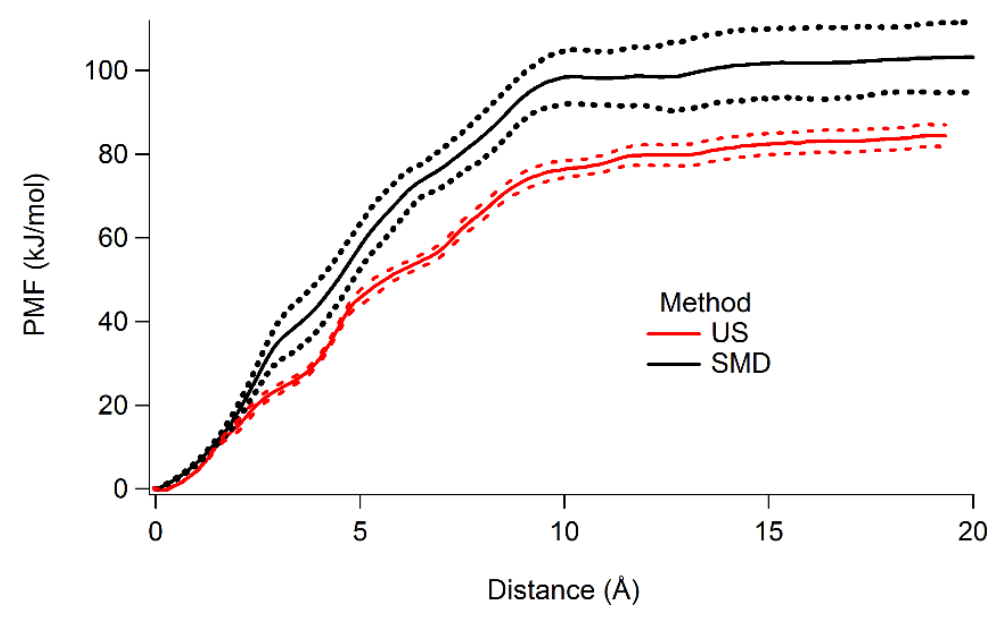

Figure 4. Comparison of the PMF calculated for SMD and US using a restraint force constant of $1000 \mathrm{~kJ} /\left(\mathrm{mol} \mathrm{nm}{ }^{2}\right)$. The error bars are shown as dashed lines above and below the calculated PMF representing the standard deviations from the average of 100 trajectories. 


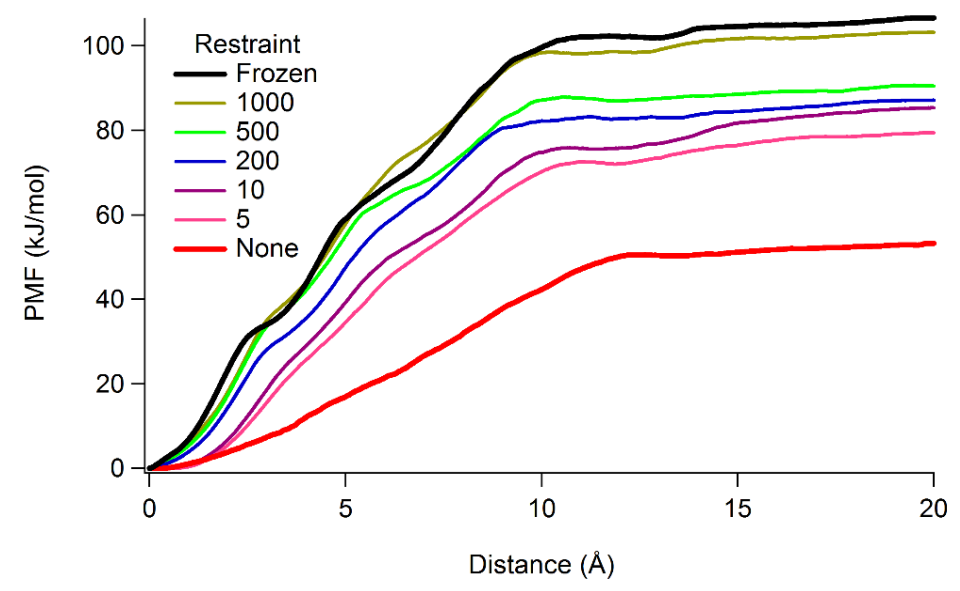

Figure 5. PMF for pulling simulation of protein and inhibitor (DHP + 4-BP) for various values of the backbone restraint harmonic potential shown in the legend $(5,10$, 200, 500 and $1000 \mathrm{~kJ} /\left(\mathrm{mol} \mathrm{m^{2 }}\right)$. The frozen and completely unrestrained backbone are shown as the black and red curve, respectively. These PMFs are the result of an average of 100 trajectories, with the exception of the frozen backbone (10 averaged trajectories) and the unrestrained PMF (200 averaged trajectories). 

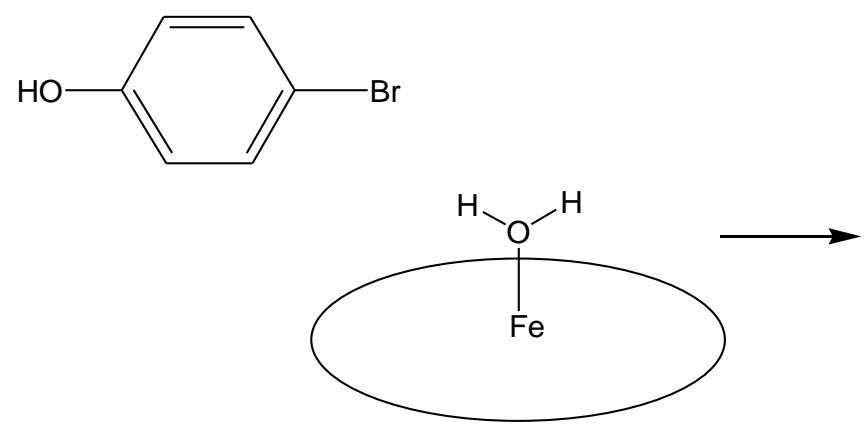

$\mathrm{H}_{\mathrm{O}^{-}} \mathrm{H}^{-1}$

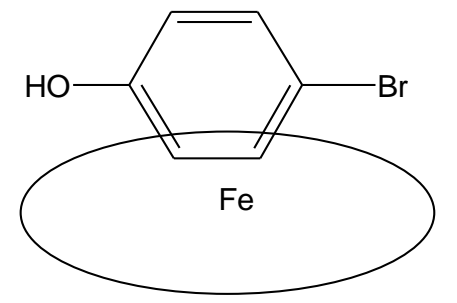

Scheme I. The binding of the inhibitor in an internal cavity in a conformation perpendicular to the heme depicts the coupling of the binding of the inhibitor to the displacement of an $\mathrm{H}_{2} \mathrm{O}$ molecule from the heme $\mathrm{Fe}$ atom. 